\title{
A new input-based event-triggered control of a discrete-time singularly perturbed system under a resource constraint environment
}

\author{
Poonam Sahu, Manisha Bhandari and Deepak M. Fulwani Member, IEEE
}

\begin{abstract}
This paper proposes a new input-based eventtriggered control for a discrete-time singularly perturbed system, where latest measurements are transmitted when a pre-defined condition is satisfied on input. The other significant contribution of the proposed work is a separation of control updates for slow and fast dynamics. The proposed event-triggered control paradigm updates and broadcasts the current measurements of slow and fast dynamics in their respective time scales. Furthermore, the ultimate boundedness of the system states is proved using ISS Lyapunov function. A numerical example is presented to show the superiority of the proposed work over the conventional periodic controller and state-based event-triggered controller.
\end{abstract}

Index Terms-Singularly perturbed systems (SPSs), Eventtriggered control (ETC), Event-triggered mechanism (ETM).

\section{INTRODUCTION}

$\mathbf{F}$ OR more than a decade, event-triggered control (ETC) [1]-[3] has been explored extensively in the control literature. The seminal concept of ETC is that the broadcast of current measurements of the system and updates of the control law can be delayed until a pre-designed condition on system states is satisfied [1]-[5]. ETC reduces communication and allows regulated use of resources vis-a-vis the conventional periodic controller. The saving of resources is especially important for the resource constraint environment such as NCS.

Physical systems that have distinct fast and slow dynamics evolving together are often represented as SPSs [6]-[8]. The simultaneous presence of fast and slow dynamics results in a widely separated set of eigenvalues. This causes numerical stiffness in system equations, which poses a significant challenge that cannot be easily dealt with by the conventional control methodologies. Therefore, to deal with this interactive phenomenon in SPSs, the control-law is designed separately in slow and fast time scales known as composite control. The composite control approach has been addressed widely in the literature for continuous or periodic feedback [7] [9]. However, when it comes to deal with the constrained resources, a limited number of results are available, where ETC has been considered for the SPSs (see [4], [5], [10], [11]). In [10], a stabilized ETC is proposed for nonlinear SPSs, where only slow variables are considered. In [5], discrete ETC is proposed for SPSs, where fast and slow

P. Sahu and D. M. Fulwani are with the Electrical Department, Indian Institute of Technology Jodhpur, 342037, Rajasthan, India (e-mail: sahu.6@iitj.ac.in; e-mail:df@iitj.ac.in).

M. Bhandari is with the Department of Electronics, Rajasthan Technical University, Kota, 324007, India (e-mail: mbhandari@rtu.ac.in). states are sampled synchronously. An asynchronous statedependent ETC is proposed for continuous-time SPSs in [4] that ensures the asymptotic convergence of the system states. In [11], a robust dynamic ETC is proposed for the slow sampling model of SPSs. In case of continuous ETC [2], [4], additional computation is required to show that the closedloop system maintains satisfactory performance and excludes the Zeno phenomenon. However, the Zeno phenomenon can be automatically avoided in discrete-time systems [3], [5], [12].

It is worth noting that the majority of the works reported on ETC are state-dependent, where control law is updated when the norm of state-based error exceeds the pre-defined threshold. However, the control law $u(k)=G x(k)$ depends on states multiplying by a scaling factor $G$; therefore, it may happen that even if the input changes significantly, it is not updated until the corresponding state-based event-triggered condition is satisfied. On the other hand, it is quite possible that the change in states may not always result in a significant change in input; thus, an update of control law can be skipped. Therefore, the use of state-based error in ETC may either delay the control updates and deteriorate the performance or cause redundant updates of the control law. Hence, it is natural to consider the input-based ETC, where the control law is updated if there is a significant change in the input. In literature, a few works are reported [13]-[15], in which inputbased ETC is considered for different applications. In [13], the effectiveness of the control performance is measured by the cost function, while in [15], control performance is compared with state-based ETC using transmission rate and cost index.

The main objective of our work is to design and implement the composite control law [9] for the discrete-time SPS using input-based asynchronous ETC, where slow and fast dynamics are independently measured and are communicated when their respective ETCs are satisfied, shown in Fig. 1. In contrast to the existing results in SPSs, the main contributions of the proposed work are pointed out as follows: (i) The proposed input-based ETM updates the control law of slow and fast dynamics when needed by avoiding the redundant execution of the control task. (ii) Due to asynchronous ETMs and independent measurements of slow and fast dynamics, the usage in communication and computation are reduced significantly. (iii) Integral error norm and transmission rate are used to validate the effectiveness of the proposed ETM. (iv) Ultimate boundedness of the system states is achieved using ISS stability.

Notation \& Definitions: Subscript 's' and 'f' represent slow 


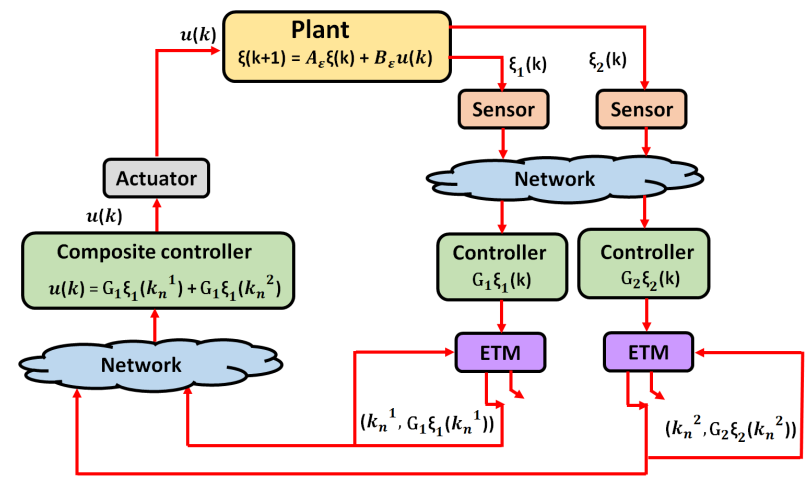

Fig. 1. Complete structure of event-triggered closed-loop system with asynchronous sampling

and fast dynamics respectively. A function $f:[0, a) \rightarrow$ $[0, \infty), a>0$ is said to be a class $K$ function, if $f$ is strictly increasing and $f(0)=0$. A function $f:[0, a) \rightarrow[0, \infty), a>$ 0 is said to be a class $K_{\infty}$ function if it belongs to class $K$, and $a \rightarrow \infty$. Also $f(m) \rightarrow \infty$ if $m \rightarrow \infty$. We are using the standard notations from [1] for sampling instants, where $k$ represents the sequence of periodic sampling instants and $k_{n},(n \in\{\mathbb{N} \cup\{0\}\})$ represents the sequence of eventoccurrence instants. However, in this paper, the sequence of event-occurrence instants for slow dynamics and fast dynamics are independently represented by $k_{n}^{1}$ and $k_{n}^{2}$ respectively.

Definition 1: Consider a discrete-time system

$$
\zeta(k+1)=A_{\epsilon} \zeta(k)+B_{\epsilon} u(k)
$$

$\zeta(k)$ and $u(k)$ represent a state vector and an input vector respectively. $A_{\epsilon}$ and $B_{\epsilon}$ denote the state matrix and an input matrix respectively. A vector function $f(k, \epsilon)$ is said to be an $O(\epsilon)$ over an interval $\left[k_{1}, k_{2}\right]$ if there exist positive constants $c$ and $\epsilon^{*}$ such that $\|f(k, \epsilon)\| \leq k \epsilon, \forall \epsilon \in\left[0, \epsilon^{*}\right], \forall k \in\left[k_{1}, k_{2}\right]$. See [9].

Definition 2: [16] Consider a discrete-time system (1), a positive function $V(\zeta(k))$ is said to be an ISS Lyapunov function if it satisfies the following.

$$
\alpha_{1}(\|\zeta(k)\|) \leq V(\zeta(k)) \leq \alpha_{2}(\|\zeta(k)\|)
$$

where, $\alpha_{1}, \alpha_{2}$ are the class $K_{\infty}$ functions, and

$$
V(\zeta(k)) \leq-\beta(\|\zeta(k)\|)+\gamma(\|u(k)\|)
$$

where, $\beta, \gamma$ are the class $K_{\infty}$ and class $K$ functions respectively.

Lemma 1: [17] For any two matrices $X_{1}, X_{2}$ and a scalar $\theta>0$, we can write $2 X_{1} X_{2} \leq \theta X_{1}^{T} X_{1}+\frac{1}{\theta} X_{2}^{T} X_{2}$.

\section{SYSTEM DESCRIPTION}

Consider a class of linear discrete-time SPS as follows.

$$
\left[\begin{array}{c}
\zeta_{1}(k+1) \\
\zeta_{2}(k+1)
\end{array}\right]=\left[\begin{array}{cc}
I_{n}+\epsilon A_{11} & \epsilon A_{12} \\
A_{21} & A_{22}
\end{array}\right]\left[\begin{array}{l}
\zeta_{1}(k) \\
\zeta_{2}(k)
\end{array}\right]+\left[\begin{array}{c}
\epsilon B_{1} \\
B_{2}
\end{array}\right] u(k)
$$

which is further represented in a compact form as (1) with $\zeta(k):=\left[\begin{array}{c}\zeta_{1}(k) \\ \zeta_{2}(k)\end{array}\right], A_{\epsilon}:=\left[\begin{array}{cc}I_{n}+\epsilon A_{11} & \epsilon A_{12} \\ A_{21} & A_{22}\end{array}\right], B_{\epsilon}:=\left[\begin{array}{c}\epsilon B_{1} \\ B_{2}\end{array}\right]$ $\zeta_{1}(k) \in \mathbb{R}^{n}$ and $\zeta_{2}(k) \in \mathbb{R}^{m}$ represent the slow state vector and fast state vector with initial conditions $\zeta_{1}(k=0)=\zeta_{1}^{0}$ and $\zeta_{2}(k=0)=\zeta_{2}^{0}$ respectively. Input vector is represented by $u(k)$. $I_{n}$ represents the identity matrix of $n \times n$ dimensions. $A_{11}, A_{11}, A_{11}, A_{11}$ represent the known constant matrices of suitable dimensions. $I_{n}+\epsilon A_{11}$ denotes the slow subsystem matrix whose eigenvalues are located near to the unit circle. $A_{22}$ is the fast subsystem matrix whose eigenvalues are placed closer to the origin.

$\epsilon$ is the small positive scalar that is used to separate the slow and fast dynamics of the original system (4). The presence of singular perturbation parameter $\epsilon$ leads to a huge computational complexity. Therefore, to alleviate the computational burden caused due to SPP $(\epsilon)$, the system in (4) is reduced to two lower dimensional subsystems in two different time scales using [7], [9] and then the control law for the system (4) is obtained in the composite form of two controllers of the lower order subsystems as follows.

1) Slow subsystem: The slow subsystem can be defined with the assumption that the fast transients have reached their steady state and; therefore, the effect of fast dynamics is negligible once their transients die out. Thus, replacing the slow dynamics by its steady-state equation, the slow part of $\zeta_{2}(k)$ in (4) can be described by

$\zeta_{2 s}(k)=\left(I_{m}-A_{22}\right)^{-1}\left[A_{21} \zeta_{1 s}(k)+A_{22} \zeta_{2 s}(k)+B_{2} u_{s}(k)\right]$

where, $\left(I_{m}-A_{22}\right)^{-1}$ is assumed to be non-singular. Substituting the slow dynamics (6) in the system, we can obtain the slow subsystem as

$$
\begin{gathered}
\zeta_{1 s}(k+1)=\left(I_{n}+\epsilon A_{s}\right) \zeta_{1 s}(k)+\epsilon B_{s} u_{s}(k) \\
A_{s}:=A_{11}+A_{12}\left[I_{m}-A_{22}\right]^{-1} A_{21} \\
B_{s}:=B_{1}+A_{12}\left[I_{m}-A_{22}\right]^{-1} B_{2}
\end{gathered}
$$

Initial condition of slow states is denoted by $\zeta_{1 s}(0)=\zeta_{1}(0)$.

2) Fast subsystem: Similarly, the fast dynamics are obtained by considering the slow variables constant during the fast transients, i.e., $\zeta_{1}(k)=\zeta_{1 s}(k)$ and by defining the fast variables

$$
\zeta_{2 f}(k):=\zeta_{2}(k)-\zeta_{2 s}(k), u_{f}(k):=u(k)-u_{s}(k)
$$

the fast subsystem of (4) becomes

$$
\begin{gathered}
\zeta_{2 f}(k+1)=A_{f} \zeta_{2 f}(k)+B_{f} u_{f}(k) \\
A_{f}:=A_{22}, B_{f}:=B_{2}
\end{gathered}
$$

The initial condition is represented by $\zeta_{2 f}(0)=\zeta_{2}(0)-\zeta_{2 s}(0)$.

3) Composite control:

Assumption 1: The pair $\left(I_{n}+\epsilon A_{s}, \epsilon B_{s}\right)$ and $\left(A_{f}, B_{f}\right)$ are stabilizable.

Assumption 2: There exist matrices $G_{s}, G_{2}$ of appropriate dimensions such that $I_{n}+\epsilon\left(A_{S}+B_{S} G_{S}\right)$ and $A_{22}+B_{2} G_{2}$ are schur stable.

Under the Assumption 1 and Assumption 2, we can design a stabilized composite controller in terms of the controller corresponding to the reduced-order subsystems (7) and (10) as

$$
u(k)=u_{s}(k)+u_{f}(k)=G_{1} \zeta_{1}(k)+G_{2} \zeta_{2}(k)
$$




\section{MAIN RESULTS}

The first part of this section proposes a new input-based ETM for the discrete-time SPS (4). In the second part, we obtain the closed-loop system with the event-triggered input, while the last part of this section establishes the ultimate boundedness of the system states in (4) using ISS criteria.

\section{A. Event-triggered mechanism}

The main objectives of the proposed ETM are pointed out as follows:

1) To avoid the redundant update of control law of slow and fast subsystems by considering an input-based error in the triggered condition.

2) Separate event-triggered conditions are designed, as shown in Fig. 1, by leveraging the intrinsic properties of slow and fast dynamics leading to asynchronous data transmission.

The sequence of triggering instances for both the slow and fast dynamics are independently determined when their corresponding triggered conditions are satisfied. Error at each triggering instant is zero. At sampling instants between two events, error for both the slow and fast dynamics are defined by

$$
e_{i}(k):=G_{i} \zeta_{i}\left(k_{n}^{i}\right)-G_{i} \zeta_{i}(k), k \in\left(k_{n}^{i}, k_{n+1}^{i}\right)
$$

$k$ represents the sequence of discrete sampling instants while the sequence of triggering instants are represented by $k_{n}^{i} . i=1$ represents the slow subsystem and $i=2$ represents the fast subsystem. A new event is triggered and the corresponding control law is updated if the following condition is satisfied.

$$
k_{n+1}^{i}=\inf \left\{k: k>k_{n}^{i},\left\|e_{i}(k)\right\|-\sigma_{i}\left(a_{0}+c_{1}^{k}\right)>0\right\}
$$

where, $0 \leq a_{0}, c_{1}<1$, and $\sigma_{i}\left(0<\sigma_{i}<1\right)$ are the design parameters. Both $a_{0}$, and $c_{1}$ can not be zero at the same time. We consider the time-dependent threshold from [18], where event-triggered error is the function of system states.

\section{$B$. Event-triggered closed-loop system}

The original SPS is separated into two slow and fast dynamics, as shown in Fig. 1, which have their respective sensors and controllers and ETMs. After the occurrence of an event, each subsystem sends its respective control signal to the actuator through the network. The actuator uses the composite control law by adding slow and fast input and sends it to the original plant. Consider the latest triggering instants of slow and fast dynamics as $k_{n}^{1}$ and $k_{n}^{2}$ and next triggering instants at which event-triggered condition corresponding to the slow and fast dynamics are satisfied as $k_{n+1}^{1}$ and $k_{n+1}^{2}$. We obtain the composite controller (12) with event-triggered input as

$$
u(k)=G_{1} \zeta_{1}\left(k_{n}^{1}\right)+G_{2} \zeta_{2}\left(k_{n}^{2}\right)
$$

where, $G_{1} \zeta_{1}\left(k_{n}^{1}\right)$ remains same $\forall k \in\left[k_{n}^{1}, k_{n+1}^{1}\right)$ and $G_{2} \zeta_{2}\left(k_{n}^{2}\right)$ remains same $\forall k \in\left[k_{n}^{2}, k_{n+1}^{2}\right)$. Rewriting (4) using (15) and (13), we obtain

$$
\begin{aligned}
\zeta_{1}(k+1) & =\left(I_{n}+\epsilon A_{11}\right) \zeta_{1}(k)+\epsilon A_{12} \zeta_{2}(k) \\
& +\epsilon B_{1}\left(G_{1} \zeta_{1}\left(k_{n}^{1}\right)+G_{2} \zeta_{2}\left(k_{n}^{2}\right)\right) \\
\zeta_{2}(k+1) & =A_{21} \zeta_{1}(k)+A_{22} \zeta_{2}(k) \\
& +B_{2}\left(G_{1} \zeta_{1}\left(k_{n}^{1}\right)+G_{2} \zeta_{2}\left(k_{n}^{2}\right)\right)
\end{aligned}
$$

(16) is further simplified into

$$
\begin{aligned}
& \zeta_{1}(k+1)=M_{11} \zeta_{1}(k)+M_{12} \zeta_{2}(k)+\epsilon B_{1} e_{1}(k)+\epsilon B_{1} e_{2}(k) \\
& \zeta_{2}(k+1)=M_{21} \zeta_{1}(k)+M_{22} \zeta_{2}(k)+B_{2} e_{1}(k)+B_{2} e_{2}(k)
\end{aligned}
$$

where, $M_{11}:=I_{n}+\epsilon A_{11}+\epsilon B_{1} G_{1}, M_{12}:=\epsilon A_{12}+\epsilon B_{1} G_{2}$, $M_{21}:=A_{21}+B_{2} G_{1}, M_{22}:=A_{22}+B_{2} G_{2}, e(k):=e_{1}(k)+$ $e_{2}(k)$.

C. Separation of slow and fast dynamics from the eventtriggered closed-loop system

Transformation matrix $T$ is used to separate the slow and fast dynamics of (17), where $T$ is assumed to be non-singular. Define

$$
\left[\begin{array}{l}
z_{1}(k) \\
z_{2}(k)
\end{array}\right]:=T\left[\begin{array}{l}
\zeta_{1}(k) \\
\zeta_{2}(k)
\end{array}\right], T:=\left[\begin{array}{cc}
I_{n}-\epsilon S V & -\epsilon S \\
V & I_{m}
\end{array}\right]
$$

Matrix $S \in \mathbb{R}^{m \times n}$ and matrix $V \in \mathbb{R}^{n \times m}$ are the solutions of the following equations.

$$
\begin{aligned}
& M_{21}-M_{22} S+\epsilon S M_{11}-\epsilon S M_{12} S+S=0 \\
& M_{12}-V M_{22}+\epsilon M_{11} V-\epsilon M_{12} S V-\epsilon V S M_{12}+V=0
\end{aligned}
$$

For order $O(\epsilon)$ approximations, the system (17) is represented in terms of its decoupled form as

$$
\begin{gathered}
z(k+1)=M z(k)+B e(k) \\
z(k):=\left[\begin{array}{c}
z_{1}(k) \\
z_{2}(k)
\end{array}\right], B:=\left[\begin{array}{c}
\epsilon\left(B_{1}-S B_{2}\right) \\
B_{2}
\end{array}\right]+O(\epsilon), \\
M:=\left[\begin{array}{cc}
M_{s}+O(\epsilon) & O \\
O & M_{f}+O(\epsilon)
\end{array}\right]
\end{gathered}
$$

where, ' $O^{\prime}$ denotes the zero matrix of suitable dimensions. $M_{s}:=\left(I+\epsilon\left(A_{s}+B_{s} G_{1}\right)\right), M_{f}:=A_{f}+B_{f} G_{2}$. Since the states in (20) are the transformed matrix of the system (17). Therefore, stability of system (20) implies the stability for the system original system (17).

\section{System stability}

Theorem 1: Consider the event-triggered closed loop system (17) with triggering rule (14), the state trajectory $\zeta(k)$ of the system (17) are uniformly ultimate bounded with radius $(r)$ as

$$
\begin{gathered}
r=\|T\|^{-1} \beta \sigma a_{0} \\
\beta:=\left(\frac{\left\|M^{T} P B\right\|^{2}}{\lambda_{\min }^{2}(Q)}+\frac{\left\|B^{T} P B\right\|}{\lambda_{\min }(Q)}\right)^{\frac{1}{2}}
\end{gathered}
$$

$\lambda_{\min }(Q)$ is the minimum eigenvalue of the matrix $Q . T$ is the transformed matrix defined in (18).

Proof 1: By design, $A_{22}+B_{2} F_{2}$ and $\left(I_{n}+\epsilon\left(A_{s}+B_{s} G_{1}\right)\right)$ are stable matrices. Therefore, $M_{s}$ and $M_{f}$ are also stable. For the given matrices $Q>0$, there exists positive definite matrix $P>0$ which is the unique solution of the following Ricatti equation.

$$
M^{T} P M-P=-Q
$$

Consider an ISS Lyapunov candidate function $V(k, z)$ as

$$
V(k, z)=z(k)^{T} P z(k)
$$


which can be further bounded as

$$
\lambda_{\min }(P)\|z(k)\|^{2} \leq z(k)^{T} P z(k) \leq \lambda_{\max }(P)\|z(k)\|^{2}
$$

$\lambda_{\min }(P)$ and $\lambda_{\max }(P)$ represent the minimum and maximum eigenvalues of $P$. Computing the forward difference $\Delta V=$ $V(k+1, z)-V(k, z)$, we have

$$
\Delta V=z^{T}(k+1) P z(k+1)-z^{T}(k) P z(k)
$$

$\Delta V$ in (27) is written by using (20).

$$
\begin{aligned}
\Delta V & =(M z(k)+B e(k))^{T} P(M z(k)+B e(k)) \\
& -z(k)^{T} P z(k)
\end{aligned}
$$

which is further simplified using (24) as

$$
\begin{aligned}
& \Delta V=z(k)^{T}(-Q) z(k)+z(k)^{T} M^{T} P B e(k) \\
& +e(k)^{T} B^{T} P M z(k)+e(k)^{T} B^{T} P B e(k)
\end{aligned}
$$

Using Lemma 1 and (26), $\Delta V$ in (29) is bounded by

$$
\begin{aligned}
& \Delta V \leq-\|z(k)\|^{2} \lambda_{\min }(Q)+\theta\|z(k)\|^{2} \\
& +\frac{1}{\theta}\left\|M^{T} P B\right\|^{2}\|e(k)\|^{2}+\|e(k)\|^{2}\left\|B^{T} P B\right\|
\end{aligned}
$$

$\theta>0$ is an arbitrary scalar, thus substituting this by $\frac{\lambda_{\min }(Q)}{2}$, We can write the inequality (30) as

$$
\begin{aligned}
& \Delta V \leq-\frac{\lambda_{\min }(Q)}{2}\|z(k)\|^{2}+\frac{2\left\|M^{T} P B\right\|^{2}}{\lambda_{\min }(Q)}\|e(k)\|^{2} \\
& +\|e(k)\|^{2}\left\|B^{T} P B\right\|
\end{aligned}
$$

Denoting

$$
\begin{aligned}
\alpha_{3} & :=\frac{\lambda_{\min }(Q)}{2} \\
\gamma_{1} & :=\left(\frac{2\left\|M^{T} P B\right\|^{2}}{\lambda_{\min }(Q)}+\left\|B^{T} P B\right\|\right)
\end{aligned}
$$

We can rewrite (31) as

$$
\Delta V \leq-\alpha_{3}\|z(k)\|^{2}+\gamma_{1}\|e(k)\|^{2}
$$

$\Delta V \leq 0$ if the following inequality holds.

$$
\|z(k)\| \geq\left(\frac{\left\|M^{T} P B\right\|^{2}}{\lambda_{\min }^{2}(Q)}+\frac{\left\|B^{T} P B\right\|}{\lambda_{\min }(Q)}\right)^{\frac{1}{2}}\|e(k)\|
$$

Using (23), (34) is expressed as

$$
\|z(k)\| \geq \beta\|e(k)\|
$$

In view of the event-triggered condition (14), $\|e(k)\|$ in (35) is substituted by $\sigma\left(c_{1}^{k}+a_{0}\right)$, where $\sigma=\sqrt{\sigma_{1}^{2}+\sigma_{2}^{2}}$. Hence, $\Delta V \leq 0$, for the region

$$
\|z(k)\| \geq \beta \sigma\left(c_{1}^{k}+a_{0}\right)
$$

For $k \rightarrow \infty, c_{1}^{k}=0$. Therefore, the bounded regions of $z(k)$ is given by

$$
\|z(k)\| \geq \beta \sigma a_{0}
$$

Now using (37) and the transformation matrix considered in (18), we can obtain the ultimate bound for the system states (4) as

$$
\|\zeta(k)\| \geq\|T\|^{-1} \beta \sigma a_{0}
$$

which implies that the state trajectories of $\zeta(k)$ will enter in an ellipsoid which has center at origin and have radius $\|T\|^{-1} \beta \sigma a_{0}$. This completes the proof.

\section{Simulation Results}

Consider the discrete-time SPS given by (1) with $A_{\epsilon}=$ $\left[\begin{array}{cc}1-\epsilon & 0.1 \epsilon \\ 0.15 & 0.1\end{array}\right], B_{\epsilon}=\left[\begin{array}{c}2 \epsilon \\ -0.1\end{array}\right]$. Feedback gain $G_{1}$ and $G_{2}$ are calculated using pole-placement technique as $0.1034,-0.25$ respectively for $\epsilon=0.02$. Initial conditions are $\zeta(0)=$ $\left[\begin{array}{ll}0.05 & 0.01\end{array}\right]^{T}$. The design parameters $\sigma_{1}$ and $\sigma_{2}$ are fixed at 0.01. Simulation is performed for different values of design parameters. Fig. 2 shows the convergence of slow and fast states of the system. Fig. 3 and Fig. 4 depict the composite control input $u(k)$ of the system and number of events for different values of $a_{0}$ and $c_{1}$ respectively. To compare the proposed ETM (14) with the state-based ETM [19], transmission rate and integral norm error such as mean square error (MSE) and Integral of square time derivative of the control input (ISTC) [20] are considered which are defined as

$$
\begin{gathered}
T_{r}=\frac{\text { Number of control updates for a given run time }}{\text { Total number of periodic samples }} \\
M S E=\frac{1}{N} \sum_{k=1}^{k-N}\|e(k)\|^{2}, I S T C=\frac{1}{\Delta t} \sum_{k=1}^{k=N}(\Delta u)^{2}
\end{gathered}
$$

$N$ denotes the total number of samples. $\Delta t$ is the time interval. $\Delta u$ is the first derivative of the current input. By letting the simulation run for $10 \mathrm{~s}$ with a sampling period of $0.05 \mathrm{~s}$, the total number of periodic samples becomes 200. In periodic sampling, the measurements are transmitted, and the control law is updated at each sampling instant; therefore, in this case, transmission rate $T_{r}=100 \%$. For the synchronous ETM, transmission rates of slow and fast dynamics are $38.5 \%$ for $a_{0}=0.002, c_{1}=0,42 \%$ for $a_{0}=0, c_{1}=0.3$ and $18 \%$ for the design parameters $a_{0}=0.003$ and $c_{1}=0.01$, respectively. Table II shows the transmission rate of slow and fast dynamics of the proposed ETM for different sets of $a_{0}, c_{1}$, where, $T_{r s}, T_{r f}$ represent the transmission rates of the slow and fast dynamics, respectively. Since for large $k, c_{1}^{k}=0$; therefore, after a certain time, the control law is updated at each instant, which leads to a high transmission rate. Also, due to an asynchronous sampling, communication frequency of the system states (4) is reduced in comparison with the synchronous ETM and conventional periodic controller. From Table I and Table II, it can be noticed that ISTC and MSE are less in the case of the state-based ETC [19]. On the other hand, the transmission rate increases with the state-based ETC for the given values of design parameters. Therefore, the proposed ETC shows its advantage in reducing communication frequency over state-based ETC [19] for the given design parameters considered in Table. However, this might not be true for all the values of design parameters.

\section{CONCLUSION}

Asynchronous measurements of slow and fast dynamics have been achieved using the proposed input-based ETC. Simulation has been performed to show the effectiveness of the proposed input-based ETM by quantifying the control performance in terms of transmission rate and integral error norm. The transmission rate has been significantly reduced with the proposed ETM as compared to the periodic controller, 
TABLE I

TRANSMISSION RATE AND CONTROL PERFORMANCE WITH DESIGN PARAMETERS $\sigma_{1}=\sigma_{2}=0.1, \epsilon=0.02$ FOR THE INPUT-BASED ETM PROPOSED IN (14)

\begin{tabular}{|l|l|l|l|l|l|}
\hline$a_{0}$ & $c_{1}$ & $T_{r s}$ in $\%$ & $T_{r f}$ in $\%$ & MSE & ISTC \\
\hline 0.003 & 0.01 & 6 & 21 & $2.5 \times 10^{-3}$ & $1.2 \times 10^{-4}$ \\
0.002 & 0 & 11.5 & 38.5 & $2.6 \times 10^{-3}$ & $1.4 \times 10^{-4}$ \\
0 & 0.3 & 25 & 42 & $2.4 \times 10^{-3}$ & $1.2 \times 10^{-4}$ \\
\hline
\end{tabular}

TABLE II

TRANSMISSION RATE AND THE CONTROL PERFORMANCE WITH DESIGN PARAMETERS $\sigma_{1}=\sigma_{2}=0.1, \epsilon=0.02$ FOR THE STATE-BASED ETM PROPOSED IN [19]

\begin{tabular}{|l|l|l|l|l|l|}
\hline$a_{0}$ & $c_{1}$ & $T_{r s}$ in $\%$ & $T_{r f}$ in $\%$ & MSE & ISTC \\
\hline 0.003 & 0.01 & 23.5 & 36 & $2.3 \times 10^{-5}$ & $1.1 \times 10^{-5}$ \\
0.002 & 0 & 40 & 60 & $2.3 \times 10^{-3}$ & $1.3 \times 10^{-5}$ \\
0 & 0.3 & 78.5 & 81 & $2.2 \times 10^{-7}$ & $1 \times 10^{-5}$ \\
\hline
\end{tabular}

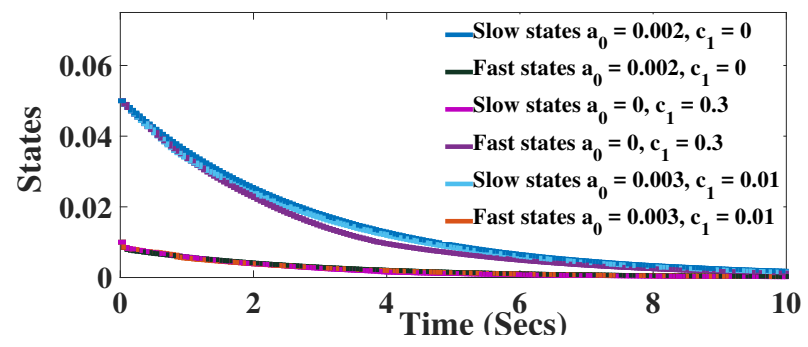

Fig. 2. Evolution of system states for different values of $a_{0}, c_{1}$ with $\sigma_{1}=$ $\sigma_{2}=0.1$

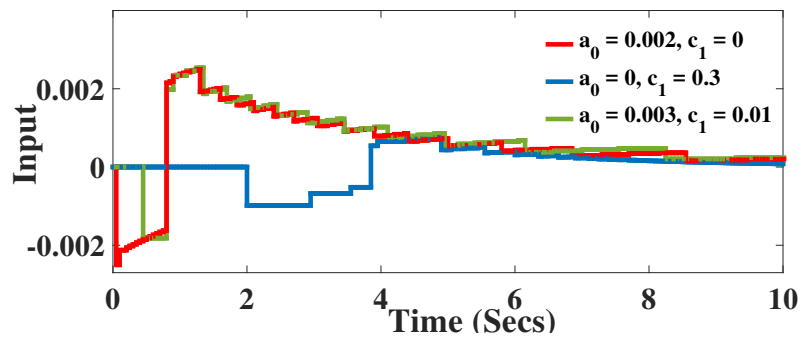

Fig. 3. Illustration of composite control law for different values of $a_{0}, c_{1}$ with $\sigma_{1}=\sigma_{2}=0.1$

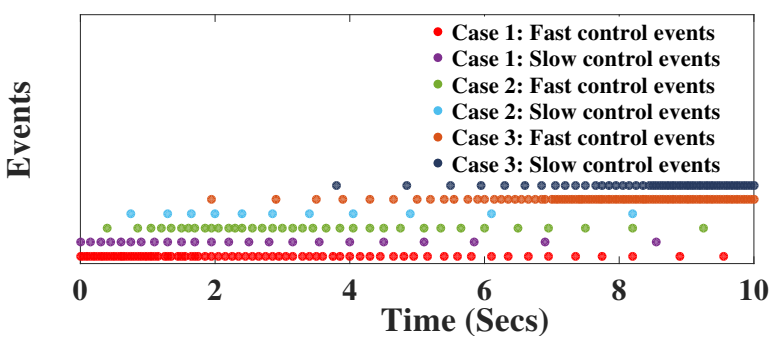

Fig. 4. Number of events for different values of $a_{0}, c_{1}$ with $\sigma_{1}=\sigma_{2}=0.1$. For case $1, a_{0}=0.002, c_{1}=0$, for case $2, a_{0}=0.003, c_{1}=0.01$ and for case $3, a_{0}=0, c_{1}=0.3$.

which has been become particularly more suitable under a resource constraint environment. Due to the input-based error, redundant updates of control law corresponding to slow and fast dynamics have been avoided while providing better control performance than the state-based ETM in terms of integral error norm (such as MSE and ISTC), and by reducing the communication frequency for the selective design parameters. Also, the asymptotic convergence of both the fast and slow dynamics of the system has been guaranteed from simulation.

\section{REFERENCES}

[1] A. Eqtami, D. V. Dimarogonas, and K. J. Kyriakopoulos, "Eventtriggered control for discrete-time systems," in Proceedings of the 2010 american control conference. IEEE, 2010, pp. 4719-4724.

[2] W. Heemels, K. H. Johansson, and P. Tabuada, "Event-triggered and self-triggered control." 2015.

[3] M. Kishida, "Event-triggered control with self-triggered sampling for discrete-time uncertain systems," IEEE Transactions on Automatic Control, vol. 64, no. 3, pp. 1273-1279, 2018.

[4] M. Bhandari, D. M. Fulwani, and R. Gupta, "Event-triggered composite control of a two time scale system," IEEE Transactions on Circuits and Systems II: Express Briefs, vol. 65, no. 4, pp. 471-475, 2018.

[5] M. S. Mahmoud, "Event-based control of discrete two-time-scale systems," in IECON 2017-43rd Annual Conference of the IEEE Industrial Electronics Society. IEEE, 2017, pp. 7217-7220.

[6] D. S. Naidu and A. J. Calise, "Singular perturbations and time scales in guidance and control of aerospace systems: a survey," Journal of Guidance, Control, and Dynamics, vol. 24, no. 6, pp. 1057-1078, 2001.

[7] P. Kokotovic, H. K. Khali, and J. O'reilly, Singular perturbation methods in control: analysis and design. Siam, 1999, vol. 25.

[8] M. S. Mahmoud, Y. Chen, and M. Singh, "A two-stage output feedback design," in IEE Proceedings D (Control Theory and Applications), vol. 133, no. 6. IET, 1986, pp. 279-284.

[9] B. Litkouhi and H. Khalil, "Multirate and composite control of two-timescale discrete-time systems," IEEE Transactions on Automatic Control, vol. 30, no. 7, pp. 645-651, 1985.

[10] M. Abdelrahim, R. Postoyan, and J. Daafouz, "Event-triggered control of nonlinear singularly perturbed systems based only on the slow dynamics," Automatica, vol. 52, pp. 15-22, 2015.

[11] J. Song and Y. Niu, "Dynamic event-triggered sliding mode control: Dealing with slow sampling singularly perturbed systems," IEEE Transactions on Circuits and Systems II: Express Briefs, 2019.

[12] W. Wu, S. Reimann, D. Görges, and S. Liu, "Suboptimal eventtriggered control for time-delayed linear systems," IEEE Transactions on Automatic Control, vol. 60, no. 5, pp. 1386-1391, 2014.

[13] B. A. Khashooei, D. J. Antunes, and W. Heemels, "Output-based eventtriggered control with performance guarantees," IEEE Transactions on Automatic Control, vol. 62, no. 7, pp. 3646-3652, 2017.

[14] G. Wei, L. Liu, L. Wang, and D. Ding, "Event-triggered control for discrete-time systems with unknown nonlinearities: an interval observerbased approach," International Journal of Systems Science, vol. 51, no. 6, pp. 1019-1031, 2020.

[15] Y. Xu, M. Fang, Z.-G. Wu, Y.-J. Pan, M. Chadli, and T. Huang, "Inputbased event-triggering consensus of multiagent systems under denial-ofservice attacks," IEEE Transactions on Systems, Man, and Cybernetics: Systems, 2018.

[16] Z.-P. Jiang and Y. Wang, "Input-to-state stability for discrete-time nonlinear systems," Automatica, vol. 37, no. 6, pp. 857-869, 2001.

[17] R. A. Horn, R. A. Horn, and C. R. Johnson, Topics in matrix analysis. Cambridge university press, 1994.

[18] M. Guinaldo, D. V. Dimarogonas, K. H. Johansson, J. Sánchez, and S. Dormido, "Distributed event-based control strategies for interconnected linear systems," IET Control Theory \& Applications, vol. 7, no. 6, pp. 877-886, 2013.

[19] P. Sahu, M. Bhandari, and D. Fulwani, "Event-trigger control of discrete two-time scale system by leveraging its intrinsic properties," in 2019 18th European Control Conference (ECC). IEEE, 2019, pp. 39703975.

[20] P. D. Domański, P. D. Domański, and Ditzinger, Control Performance Assessment: Theoretical Analyses and Industrial Practice. Springer, 2020. 